\title{
The electronics and trigger system of the High Energy Particle Detector (HEPD) onboard the China Electromagnetic Satellite (CSES)
}

\author{
V. Scotti* \\ INFN Sezione di Napoli \\ E-mail: scottivena.infn.it \\ G. Osteria \\ INFN Sezione di Napoli
}

for the Limadou-CSES Collaboration

\begin{abstract}
In this paper a description of the electronics and trigger system of the High Energy Particle Detector (HEPD) onboard the China Seismo Electromagnetic Satellite (CSES) and its main characteristics will be presented. CSES is a scientific mission dedicated to monitoring electromagnetic, plasma and particles perturbations of atmosphere and inner Van Allen belts caused by solar and terrestrial phenomena and to the study of the low energy component of the cosmic rays (5 - 100 $\mathrm{MeV}$ for electrons and $15-300 \mathrm{MeV}$ for protons). The satellite will be launched in 2017 and will host several instruments onboard: two magnetometers, an electrical field detector, a plasma analyser, a Langmuir probe and the HEPD. The HEPD, built by the Italian "Limadou" collaboration, is capable of separating electrons and protons and identify nuclei up to Iron. The HEPD comprises the following subsystems: detector, electronics, power supply and mechanics. The electronics, main focus of this paper, can be divided into three blocks: silicon detector, scintillator detectors (trigger, energy and veto detectors) and global control and data managing. The trigger system allows switching between several configurations along the orbit to cope with different fluxes encountered. Each trigger configuration corresponds to different field of view of the apparatus.
\end{abstract}

35th International Cosmic Ray Conference - ICRC2017-

10-20 July, 2017

Bexco, Busan, Korea

${ }^{*}$ Speaker. 


\section{The China Seismo-Electromagnetic Satellite}

The China Seismo-Electromagnetic Satellite (CSES), is a space mission dedicated to the monitoring of the perturbations originated by electromagnetic emissions in the atmosphere, ionosphere, magnetosphere and in the Van Allen belts [1] induced by natural sources and anthropocentric emitters. These electromagnetic anomalies from Earth and ionosphere perturbations are probably associated with earthquakes $[2,3]$.

The CSES mission aims at measuring bursts of Van Allen belt electron fluxes in the magnetosphere, possible possibly generated by an earthquake, by means of the High Energy Particle Detector (HEPD). The high inclination orbit of the satellite allows the instrument to detect particles of different nature during its revolution: galactic cosmic rays - which are modulated by the solar activity at low energies and also solar energetic particles associated to transient phenomena such as Solar Flares or Coronal Mass Ejections.

The satellite will be placed at a $98^{\circ}$ Sun-synchronous circular orbit at an altitude about 500 $\mathrm{km}$, the launch is scheduled in August 2017 with an expected lifetime of 5 years.

More details on CSES mission can be found in [6].

\section{The High Energy Particle Detector onboard CSES}

The High-Energy Particle Detector (HEPD) is one of the payloads of the CSES space mission. It has been developed by the Italian CSES collaboration.

The High Energy Particle Detector will study low energy Cosmic Rays (CR) in the energy range 3 - $300 \mathrm{MeV}$. The HEPD has to separate electrons and proton, identifying electrons within a proton background $\left(10^{-5}-10^{-3}\right)$, and identify nuclei up to Iron. The high-inclination orbit allows the telescope to detect particles of different nature during its revolution: galactic CR, Solar Energetic Particles, particles trapped in the magnetosphere. In table 1 the main parameter of the HEPD are summarized while in Figure 1 an overview of the instrument is reported.

\begin{tabular}{cc}
\hline Parameter & Value \\
\hline Energy Range & Electrons: $3-100 \mathrm{MeV}$ \\
& Protons: $30-300 \mathrm{MeV}$ \\
Angular resolution & $<8^{\circ} 5 \mathrm{MeV}$ \\
Energy resolution & $<10 \% 5 \mathrm{MeV}$ \\
Particle identification & $>90 \%$ \\
Free field of view & $\geq 70^{\circ}$ \\
Pointing & Zenith \\
Operative temperature & $-10^{\circ}+45^{\circ}$ \\
Mass & $<35 \mathrm{~kg}$ \\
Power Consumption & $<38 \mathrm{~W}$ \\
Mechanical dimensions & $20 \times 20 \times 40 \mathrm{~cm}^{3}$ \\
\hline
\end{tabular}

Table 1: HEPD main technical characteristics.

The HEPD comprises the following subsystems: detector, electronics, power supply and mechanics. The detector is contained in an aluminum box, while the electronics cards are placed 


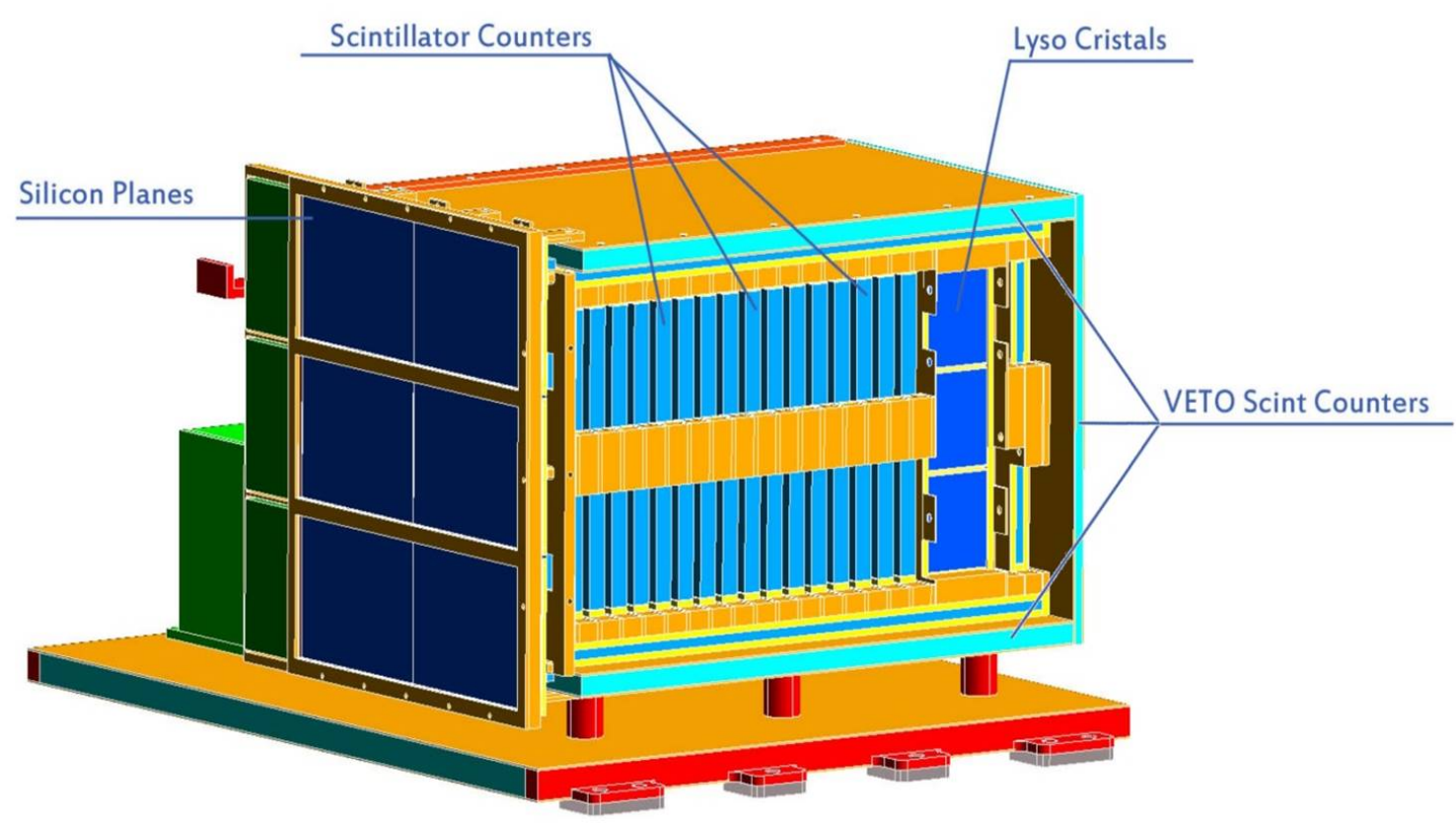

Figure 1: An overview of the HEPD instrument.

outside the detector fixed on the base plate by means of a dedicated supporting structure. The outside surface is covered with aluminized polyimide layer to assure a good thermal insulation.

The detector consists of three components:

- Silicon planes: two planes of double-side silicon micro-strip detectors are placed on the top of the detector in order to track the direction of the incident particle limiting the effect of Coulomb multiple scattering on the direction measurement;

- Trigger: a layer of thin plastic scintillator divided into six segments;

- Calorimeter: a tower of 16 layers of $1 \mathrm{~cm}$ thick plastic scintillator planes followed by a $3 \times 3$ matrix of an inorganic scintillator LYSO.

An organic scintillator is used in the calorimeter to optimize the energy resolution. In order to extend the electron measurement range to $100 \mathrm{MeV}$ an inorganic scintillator LYSO is used for the last plane of the calorimeter. The calorimeter volume is surrounded by $5 \mathrm{~mm}$ thick plastic scintillator veto planes. All the scintillator detectors (trigger, calorimeter and VETO) are read out by photomultiplier tubes (PMTs). In Figure 2 you can see a side view of the QM calorimeter which shows the plastic scintillator planes. The PMTs are at the corners of each calorimeter plane.

The good energy-loss measurement of the silicon track, combined with the energy resolution of the scintillators and calorimeter, allows identifying electrons with acceptable proton background levels $\left(10^{-5}-10^{-3}\right)$. 


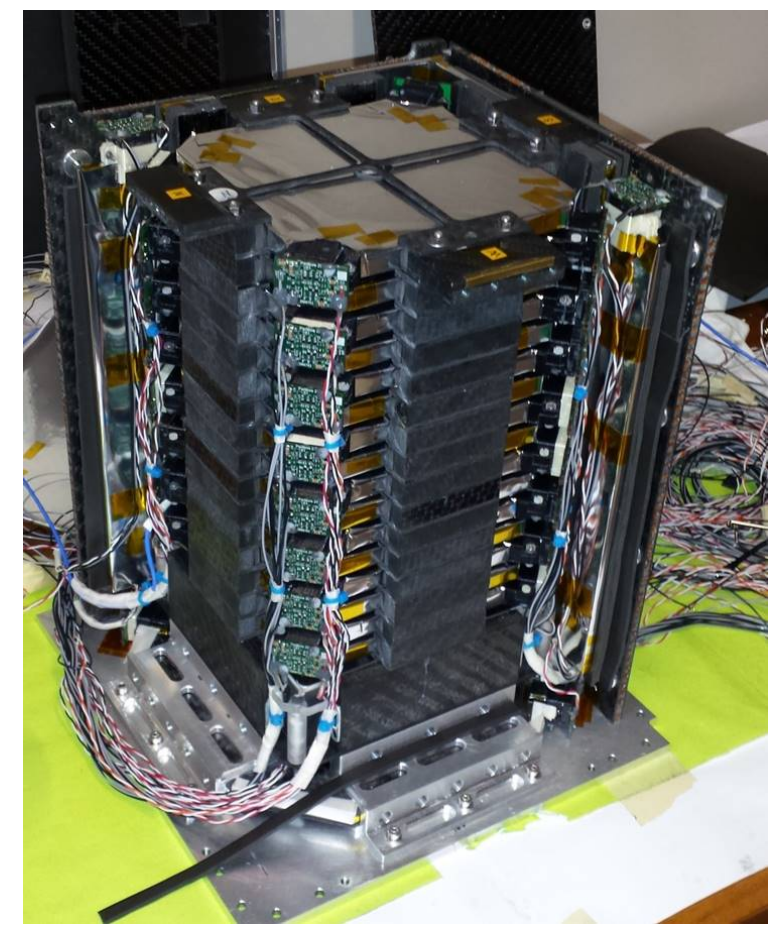

Figure 2: Side view of the QM calorimeter which shows the plastic scintillator planes. The PMTs are at the corners of each calorimeter plane.

\section{The electronics of the HEPD}

All the electronics of the HEPD is designed with embedded "Hot/Cold" redundancy and all the components of the board have been selected capable to withstand a $-40{ }^{\circ} \mathrm{C}$ to $85^{\circ} \mathrm{C}$ operating range. The trigger rate and the data stored has to cope with maximum data transfer rate from the satellite, which is $50 \mathrm{~GB}$ per day. The electronic system can be schematized into three blocks [5]:

- Silicon detector;

- Scintillator detectors (trigger, energy and veto detectors);

- Global control and data managing.

Each detector block includes power chain for bias distribution and a data acquisition processing chain. The main Power Supply provides the low voltages to the detector electronics and the high bias voltages for PMTs and silicon modules.

The position of the electronics of the HEPD with respect to the silicon detector is showed in Figure 3.

The whole electronics system is depicted in figure 4. It is composed by front-end electronics and four main boards:

- Data Acquisition (DAQ) Board: manages all the scientific data of HEPD. The DAQ accomplishes the following functionalities: acquisition of trigger signal from PMT/Trigger board, 


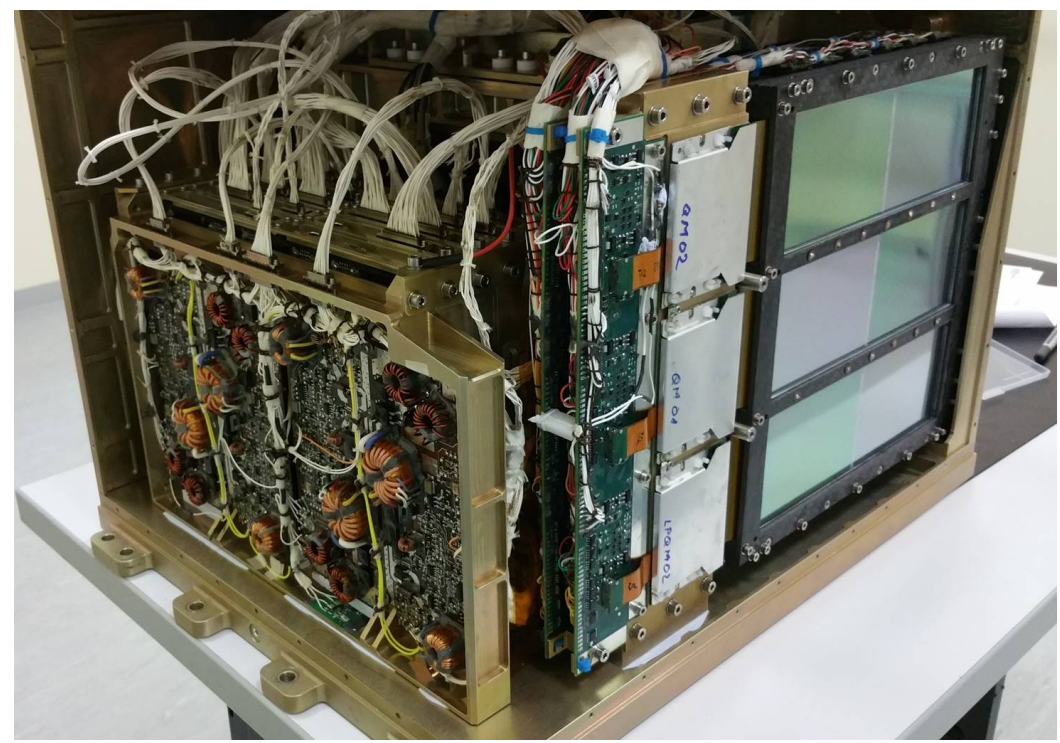

Figure 3: The electronics and the silicon detector of the HEPD.

management of hybrid circuits on the silicon planes, acquisition of silicon planes data, computing of PMTs data and silicon planes data, data compression, transmission of scientific data on the scientific data link.

- Trigger Board: manages the analog signals coming from the PMTs and generates the trigger signals needed for data acquisition. This board also generates trigger signals needed by DAQ Board to start the acquisition procedure. The main functions of the Trigger Board are to acquire the PMTs analog signal using EASIROC Integrated Circuits and to convert all the EASIROC readout signals into digital signals, to allow the DAQ board to read the EASIROC digital output, to allow the CPU to configure the EASIROC, to generate and transmit "slow" event trigger signals manipulating the "fast" trigger signals coming from EASIROC, to allow the CPU to configure the "slow" trigger generation algorithm.

- CPU Board: controls the detector and communicates with the platform of the satellite via CAN BUS interface. The CPU manages the following functionalities: communication with Satellite OBDH computer via CAN bus, storage of non volatile information, management via internal "slow" control links bus- of TM/TC and LVPS control board, Trigger Board and DAQ Board, management of system diagnostic routines and of system configuration, system monitor.

- Telemetry/Telecommand board: manages the LVPS that provides bias to the electronics subsystems, the local telemetry and telecommand signals.

All the logics has been realized with flash FPGA to improve radiation hardness.

\section{HEPD operation modes}

The HEPD-FM foresees different working modes to deal with the limited power budget, but 


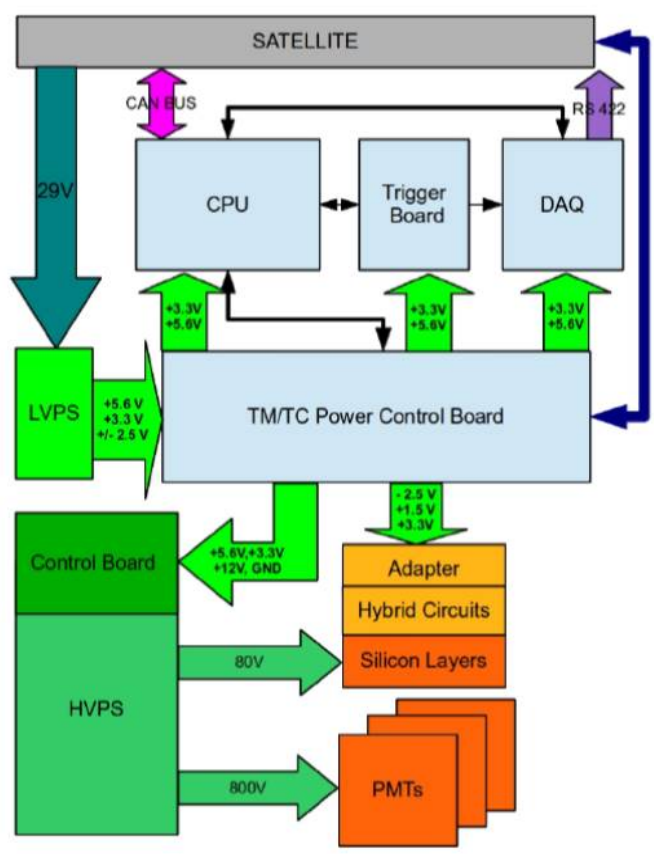

Figure 4: A block diagram of the HEPD electronics subsystem.

also whit different event rate along the satellite orbit. The functionality can be changed through the control electronics of the instrument. The main working modes are:

- Safe mode: all boards are powered on, but PMT and Si HVPSs are at a zero safe value. This mode is used at first power on.

- Stand-by mode: HEPD will be in this mode at polar latitudes $\left(65^{\circ}<\mid\right.$ lat $\left.\mid<90^{\circ}\right)$. During Stand-by, the powered boards are LVPS, TM/TC Power Control Board, HV Control Board and HVPS for Silicon Detector and CPU. The Power Consumption is stand-by mode is around $8 \mathrm{~W}(\mathrm{I}=0.270 \mathrm{~A}, \mathrm{~V}=29.5 \mathrm{~V})$.

- Nominal mode: all boards are powered on and PMTs and Silicon planes are biased at operational HV values. The operational mode can be configured in the DAQ board by 2 dedicated TCs to operate in one of the the following main operational modes:

- calibration mode: the Trigger board generates and sends to the DAQ false trigger for the calibration (of both scintillator detectors and Silicon detector). The false trigger generation foresees a check for real triggers; the false triggers should be generated in absence of a real trigger. In case of a real trigger the PMT/trigger board should wait about 2-3 microseconds (charge integration time of VAs) before generating a false trigger.

- run mode: standard run to acquire data and fake run to test the instrument.

If the instrument is in Nominal mode, when a trigger signal is generated, the Trigger Board FPGA sends the start of acquisition to each ADC. A scheme of the data acquisition procedure 
is depicted in figure 5. The analogical signal read out from the PMTs associated to scintillator detectors are transmitted directly to the Trigger Board. Signals of each data processing block related to scintillators are managed by an FPGA which issues the FAST trigger signal needed to start the acquisition of the tracker by DAQ. After an handshake protocol, if the trigger is confirmed by DAQ, the Trigger Board sends Scintillators data to DAQ Board. Scintillators and tracker data are processed by a dedicated DSP and the results are written on a DP-RAM waiting to be transferred to satellite via RS-422 on a CPU command.

During a run (normal acquisition, independently on the modes) other information are collected by the CPU board and sent via RS422. These information regard the whole run (not the single event) and are stored in run header or run tail and scientific data packets. These data regards configuration parameters of detectors and boards, broadcasts received from the satellite and relative timing information, status/error registers of each board, single PMT rate meters, temperatures and timing information.

During the startup phase (or any system reset), the Trigger Board FPGA has to configure the instrument; in this phase it sets the parameters of the detectors and Front End electronics (threshold, bias, amplifier gain, shaper time).

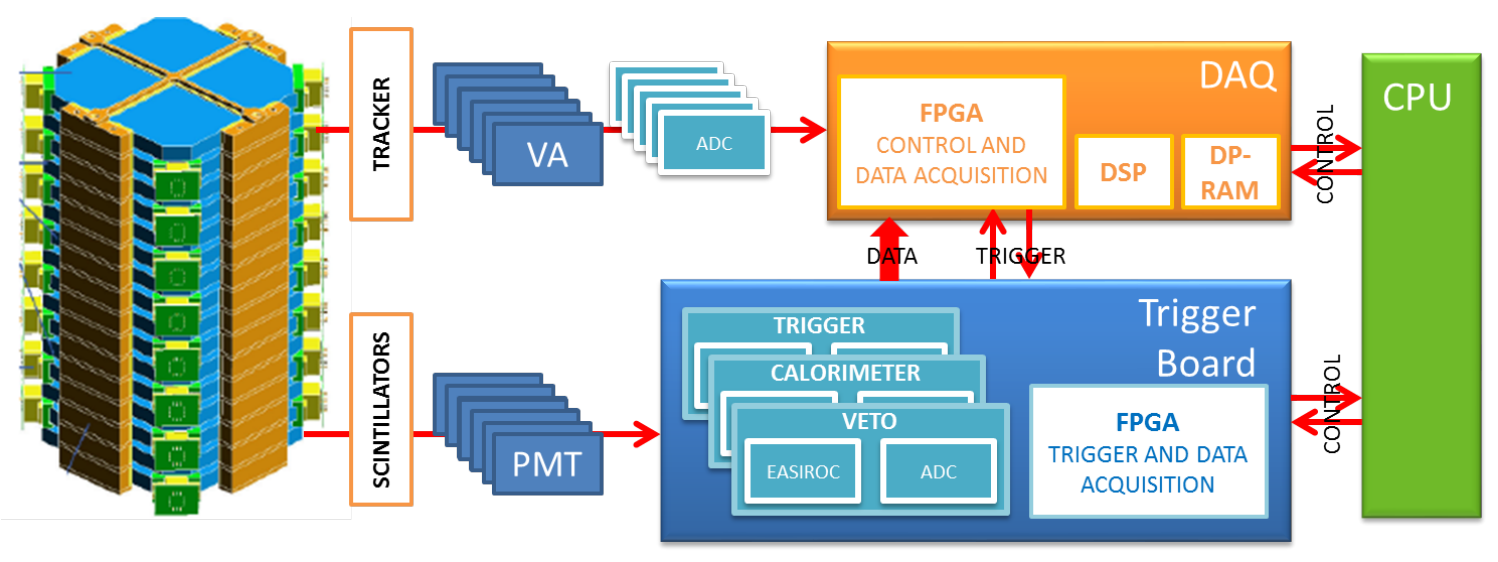

Figure 5: A scheme of the data acquisition procedure.

The Trigger board allows switching between several trigger configurations along the orbit to cope with different fluxes encountered, for example in correspondance of the South Atlantic Anomaly (SAA). In fact, the time required to readout an event in the HEPD will limit the detector operation in the SAA. A $2 \mathrm{kHz}$ event rate (500)̂ijs) limit is used to select the appropriate trigger setup. Each trigger configuration can be selected with a CPU command and a reconfiguration of the EASIROC integrated circuits. Different trigger setup corresponds to different field of view of the apparatus. In addition, the Trigger Board allows to select if the Veto is used to reduce the trigger rate and to mask trigger planes or a single PMT.

\section{Test and qualification campaign}

According to Chinese space procedures, the HEPD project involved the construction of four detector versions: the Electrical Model, the Structural and Thermal Model, the Qualification Model 
and the Flight Model. The QM and the FM of the HEPD has been built, assembled and integrated. In Spring 2016 the test and qualification campaign of the HEPD QM was started. Vibration test were held at SERMS laboratory in Terni (PG) to simulate launch and flight impact on the detector, while thermal and vacuum test taken at SERMS laboratory were used to simulate the functionality in space environment.

Finally, beam test were carried out at Beam Test Facility of the "Laboratori Nazionali di Frascati" of INFN (Italy). The detector was irradiated with electrons and positrons from 30 to 150 MeVat different angles and positions. The objective was to study the instrument response to electrons in the energy range of interest and to perform precise calibration of the calorimeter energy measurement. The FM went under the same test, in addition, the FM was irradiated with protons at the Proton Cyclotron of Trento (Italy) in the energy range 37-228 MeV.

During beam test, several trigger configuration have been tested to select the ones which will be used during flight. The data analysis allowed also to tune the default parameters which the Trigger Board uses to configure EASIROC board.

Beam test results and the performance of the apparatus both on the energy reconstruction and in the lepton/hadron separation are presented in [7]. All the results showed a good behaviour and the perfect functionality of the electronics system.

\section{Summary}

Both the QM and the FM were tested with electrons and protons beams at different energies. The QM and the FM were also submitted to vibration, thermal and vacuum test. After the acceptance test campaign, all the models were shipped to China. The HEPD FM functionality was succesfully tested with its EGSE and was accepted for assembly, integration and test at satellite level. Finally, the HEPD detector was installed on CSES satellite.

In this paper the main features of the HEPD electronics were described. Currently, pre-flight test are progress in China, then in late July the launch campaign in the Gobi desert (Inner Mongolia) will start. The memorandum between Italy and China foresees also the commissioning post launch at CSES Ground Segment in Beijing and beam test of the QM after the redelivery.

\section{References}

[1] Wang L. et al., Earthq Sci (2015) 28 4, 303

[2] Zhang X. et al., Nat. Hazards Earth Syst. Sci. (2013) 13, 197

[3] Sgrigna V. et al., Journal of Atmospheric and Solar-Terrestrial Physics (2005), 671448

[4] Ricciarini S.B. et al., EPJ Web Conf. 71 (2014) DOI: 10.1051/epjconf/20147100115

[5] Scotti V., Osteria G., for the CSES-Limadou Collaboration, Instrumentation and Methods for Astrophysics, arXiv:1701.07743.

[6] Picozza P. et al., this conference, paper ID: .

[7] Panico B. et al., this conference, paper ID:. 